\title{
Tecnura
}

\section{Multichannel assignment using K-Means in cognitive radio networks}

\section{Asignación multicanall usando K-Means en redes de radlio cognitiva}

\author{
Hans Marquez¹, Camila Salgado², Cesar Hernández³
}

Fecha de recepción: 12 de septiembre de 2016

Fecha de aceptación: 15 de febrero de 2017

Cómo citar: Márquez, H.; Salgado, C.; y Hernández C. (2017). Multichannel assignment using K-Means in cognitive radio networks. Revista Tecnura, 21(52), 68-78. doi: 10.14483/udistrital.jour.tecnura.2017.2.a05

\begin{abstract}
Context: The developed scheme allows carrying out the assignment of several frequency channels (both contiguous and not contiguous) available to the secondary users that require a higher bandwidth, under an environment of equality.
\end{abstract}

Objective: The following paper develops a multichannel assignment which allows taking in a more efficient way the spectral opportunities in cognitive radio networks.

Method: The developed assignment model is composed by the K-Means algorithm, which is in charged of carrying out the grouping of channels through clusters for the best parameters, and another algorithm in charge of establishing an equal criteria for all the secondary users that wish to transmit. The reached results were evaluated with experimental spectral occupancy data taken from the GSM frequency band. The developed model was compared with the MFA-CRN algorithm.

Results: The obtained measurements correspond to the average bandwidth, the average delay, and the fairness calculation in the assignment of several channels. The developed assignment model shows an improvement in the assignment of a higher transmission average bandwidth for each secondary user while maintaining the fairness criteria in the channel assignments.

Conclusion: Despite the increasing in the number of handoffs, it is also observed that metrics such as average bandwidth, average throughput and average delay are never negatively impacted by this increase in handoffs.

Keywords: K-Means, assignment, multichannel

\section{Resumen}

Contexto: El esquema desarrollado permite realizar la asignación de varios canales de frecuencia tanto contiguos como no contiguos que se encuentren disponibles a usuarios secundarios que requieran un mayor ancho de banda, bajo un ambiente de equidad.

Objetivo: El presente trabajo desarrolla un modelo de asignación múltiple de canales que permite aprovechar de forma más eficiente las oportunidades espectrales en redes de radio cognitiva.

Método: El modelo de asignación desarrollado está compuesto por el algoritmo K-Means, encargado de realizar el agrupamiento de canales mediante clústeres para los mejores parámetros y otro encargado

1 Electronic Engineer, master in Information and Communication Sciences. Young researcher at Colciencias, Bogotá, Colombia. Contact: hans.marquez.ramos@gmail.com

2 Electronic Engineer and Telecommunications, master in Information and Communication Sciences. Researcher at Universidad ECCl. Bogotá, Colombia. Contact: Isalgadof@ecci.edu.co

3 Electronic Engineer, master in Information and Communication Sciences, doctor in Engineering. Professor at the Universidad Distrital Francisco José de Caldas. Bogotá, Colombia. Contact: cahernandezs@udistrital.edu.co 
de establecer un criterio de equidad para todos los usuarios secundarios que deseen transmitir. Los resultados alcanzados se evaluaron con datos experimentales de ocupación espectral, capturados en la banda de frecuencia GSM. El modelo desarrollado fue comparado con el algoritmo MFA-CRN.

Resultados: Las mediciones realizadas corresponden al ancho de banda promedio, el retardo promedio y el cálculo de fairness en la asignación de varios canales. El modelo desarrollado evidencia una mejora en la asignación de un mayor ancho de banda promedio de trasmisión para cada usuario secundario, manteniendo el criterio de equidad en la asignación de los canales.

Conclusión: A pesar del aumento en el número de handoffs, se observa que métricas como ancho de banda promedio, throughput promedio y retardo promedio no se ven en ningún momento impactadas de manera negativa frente a este aumento de handoffs.

Palabras clave: K-Means, asignación, multicanal

\section{INTRODUCTION}

The cognitive radio networks respond to the need of making a flexible use of the spectrum in order to optimize the spectrum resources that are scarce in the wireless networks. This can be understood as an opportunity to improve the type of service for the users of wireless technologies, who will make an opportunistic use of the available spectrum as a consequence of having a better spectral efficiency (Hernández, Páez \& Giral, 2017; Chen, Wei, Jin, \& Ge, 2013).

These spectral opportunities are variant resources in the frequency-time domain, which the secondary users (SU) can use until the primary user (PU) requires once again the portion of the spectrum that the SU are using in order not to generate interference in the transmissions. According to this restriction, it is necessary that the $\mathrm{SU}$ move to another free spectral opportunity. This process of change is called spectral handoff. (Hernández, Márquez \& Pedraza, 2017; Tragos, Zeadally, Fragkiadakis, \& Siris, 2013)

The procedures described above are based on the assignment of one single channel. However, this paper proposes to carry out a multichannel assignment for the secondary user, allowing real time applications and single-channel for the delay-tolerant applications.

Nevertheless, assigning several channels to one single Secondary User means taking into consideration a fairness criterion for the fair assignment of resources for each one of the interested SU; it also implies choosing a method which allows carrying out the selection of the best available resources for an efficient assignment of all the users. To solve this problem, it is considered the implementation of the fairness criterion to make a fair assignment of the available resources among all the secondary user, and the K-Means algorithm (Xu, Han, \& Nie, 2016) will be used to group the best available resources as a resource classification method.

The current paper is divided as follows: Section 2 presents the related work; Section 3 describes the model development; Section 4 presents the reached results; and Section 5 shows the conclusions.

\section{RELATED WORK}

The research by (Nguyen, Madhukumar, Tio, \& Ng, 2010) proposes an algorithm that uses K-Means for dynamic grouping the bands (DBC); this implementation allows a reduction in the number of erroneous sub-bands of spurious noise and, hence, reduces the global detection time. Additionally, it presents a problem regarding the local decision combination of Secondary Users. This problem is addressed with a dynamic decision algorithm based on weight that proves superior to other algorithms with the same technique. 
On the other hand, (Chen, Hou, Huang, \& Jing, 2016) presents a cooperative algorithm for CRN, which makes use of the K-Means technique to carry out an offline training and establishing a set of data for training. This set would be utilized as a basis for the stage of online classification, which carries out a comparison between the received signals against the training data; this is how it decides the channel availability. The fusion center will sequentially make the final decision according to the local detection result of the different sensors. Each sensor is responsible for the analysis of a certain period of the received signal. This algorithm differs from others because the proposed threshold for this algorithm is measured by a similarity of characteristics in multiple dimensions and not for a fixed value. The results evaluate the performance, making a comparison between different methods, and the result shows that this scheme can achieve better performance and work in a more efficient way.

(Kang, Ryu, Lee, \& Jeong, 2011) develops a description regarding the performance of the no homogeneity detector (NHD) with several normalization methods for the processing of the radar signals airborne under the heterogeneous disorder environments. Additionally, the threshold is estimated using statistical analysis as the mean, the median and the K-Means algorithm with training data in the NHD process. The data used in the threshold is included in the recalculation of the covariance matrix of the total interference whose accuracy is evaluated through the statistical test of a modified sample matrix inversion (MSMI). The projection statistics (PS) are used to compare the performance of the detection of unique and multiple objectives. The simulation results show that the algorithm for grouping K-means is a method of normalization which can ameliorate the performance of the Space-Time Adaptive Processing (STAP) in a no homogenous environment.

(McLean, Silvius, \& Hopkinson, 2013) presents an innovative model that determines the grouping configurations for different network types and in various RF environments. A software based on
K-Means developed by the University of Maryland that demonstrates the possibility of using K-Means in cognitive radio through simulations in Matlab. The configuration shows resistance to the interference. The research also proposes a new metric called Intra-Cluster Spectrum Similarity (ICSS), which evaluates the grouping effectiveness. This metric shows that the developed model has a possibility of improving the reliability in the cognitive radio networks in a dynamic spectrum environment.

The research by (Kumar, Kandpal, Jain, Gangopadhyay, \& Debnath, 2016) implements the unsupervised grouping K-Means technique in order to improve the performance of the cooperative spectrum detection in fading channels $\kappa-\mu$. The simulations take into consideration a BPSK transmission with different parameters of the system in the receiver characterization.

(Zhang, Hu, Bao, \& Wu, 2013) proposes an algorithm of a neural network for the spectrum prediction based on K-Means called K-RBF. This algorithm can predict holes in the spectrum through the historical spectrum information of the PU, improving the efficiency of the spectrum detection process. The simulation results show that this algorithm can accurately predict the access to the PU's spectrum.

(Moumena, 2015) presents a detection method based on the union of the compressed sample (CS) and the K-means algorithm to detect the presence of the interference attack signal in the spectrum based on the two proposed hypothesis by $\mathrm{Ho}$ and $\mathrm{Hi}$ (lack of interference and presence of interference). An analog information (AIC) was used in each radio receptor to carry out the compression sampling. The compressed measurements obtained after applying CS to each radio receptor are located in a dimension matrix $Y$. The obtained results show that the original method works well and has low energy use and low computational complexity.

(Xue \& Feifei, 2015) proposes a spectrum detection method based on automatic learning. This method uses a covariance matrix with the received signal samples by several antennas. Before carrying out the detection process, the cognitive radio 
(CR) will apply first the K-means grouping algorithm in order to discover the $\mathrm{PU}$ transmission patterns. Then, the supervised learning algorithm (Support Vector Machine) will train the CR and determine the PU status. This method does not need any information about the signal, the channel, or the noise power a priori. The simulations show clearly the efficacy of the proposed model.

(Lu, Zhu, Wang, \& Fattouche, 2016) proposes a probability vector of low dimension for the machines to learn based on the spectrum cooperative detection in CRN. This approach also considers the K-Means algorithm, and the support vector machine (SVM) with the probability vector. The proposed probability vector can decrease the dimension of the energy vector present of $\mathrm{N}$ dimension to two dimensions taking into account a CRN with just one PU and $\mathrm{N} \mathrm{SU}$, resulting in a similar or better accuracy in detection, a low duration of training, and a shorter classification time.

Finally, (Bajali, Nagendra, Hota, \& Raghurama, 2016) proposes the definition of archetypal clusters without supervision, where the local energy vectors are decomposed into a collection of extreme points called archetypes. This type of cluster has similarity with the K-Means method. While the latter provides an average view of the data, the former provides an extreme view. The performance of the algorithm is evaluated through detection probability and compliance with the standard IEEE 802.22 (WRAN). In the results, the proposed algorithm is compared with k-means and SVM.

\section{METHODOLOGY}

The proposed model is presented in figure 1.

\section{Defining the simulation time and the spectral occupancy data}

The model carries out the assignment of resources according to the existing demand taking into account the existing time slots for the simulation. It should be noted that each time slot has a

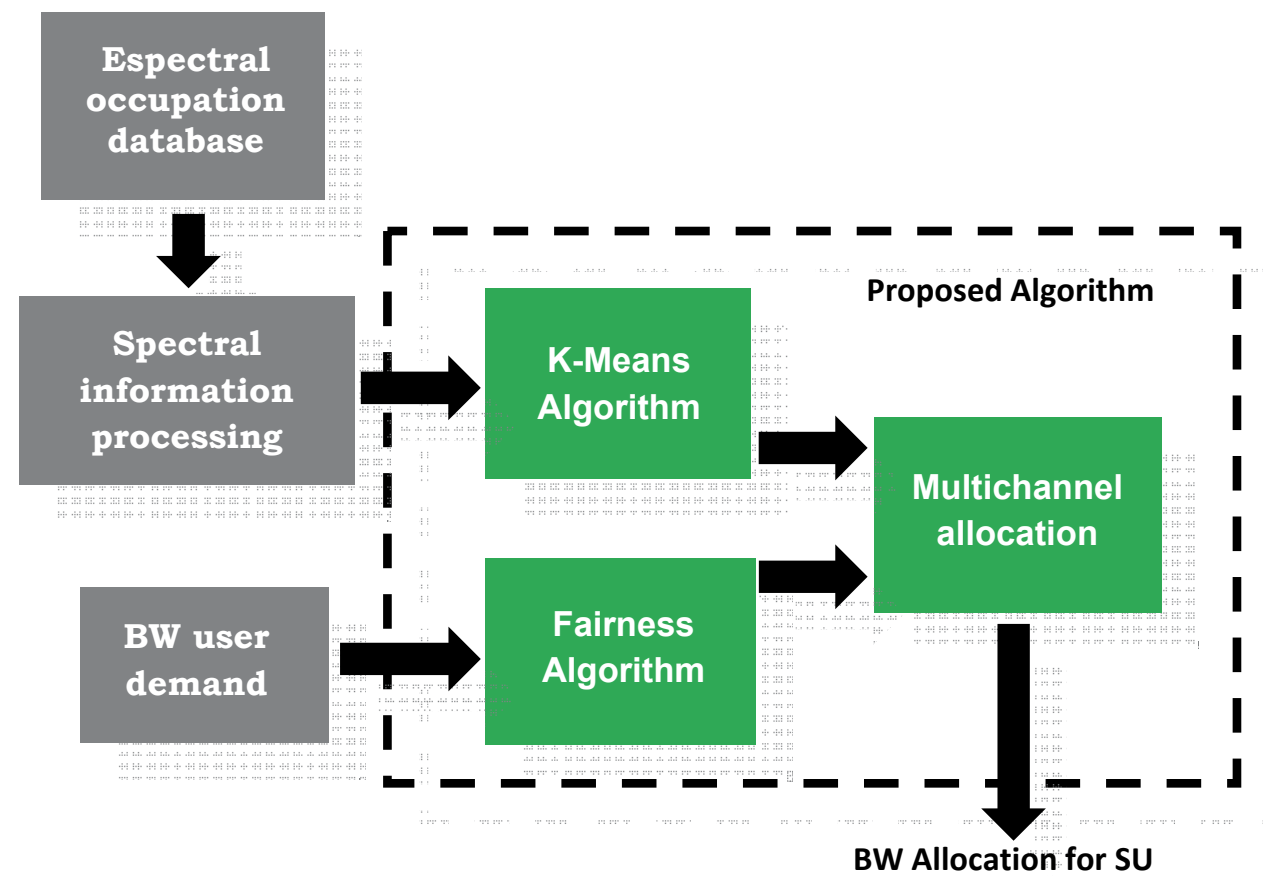

Figure 1. General structure of the proposed model

Source: own the author 
duration of $300 \mathrm{~ms}$ and the total simulation time is $10 \mathrm{~min}$.

Additionally, the model has a matrix with spectral occupancy data as input data for the GSM frequency bands making a significant differentiation in terms of measured traffic at different time intervals. Such differentiation refers to the volume of information and occupancy shown in each of the channels, which obtains high-traffic and low-traffic data. This differentiation presents the evaluation of the model in two different scenarios, each one with different characteristics.

\section{Choosing the channel selection method}

The k-means non-supervised learning algorithm is used for channel selection, which classifies data through a number $\mathrm{k}$ of clusters. Thus its goal is to define a $k$ number of centroids for each of the clusters, then it takes each of the data and associates it to the nearest center (Ramler, 2008) (Pandey, 2015). This process is carried out until all the data is assigned to one of the centroids. This procedure is performed iteratively for an n number of times; hence the centroids will vary at each iteration until they reach stability (Vassilvitskii, 2007). FinaIly, this algorithm aims to minimize an objective function known as a mean-squared error given by equation (1).

$$
J(V)=\sum^{C} \sum^{C i}\left(\left\|x_{i}-v_{j}\right\|\right)^{2}
$$

Where $x_{i}-v_{j} \|$ Is the Euclidean distance $x_{i} \mathrm{y} v_{j}, c_{i}$ Is the number of data in the cluster jth and $c$ is the centroid number.

\section{Scenario 1: System resources greater than user demands}

The resources in a system, such as bandwidth, can be used by a user who requires multi-channel transmissions. However, the condition in equation (2) must be fulfilled so the system can satisfy the demands of all users with both real-time and besteffort applications:

$$
\sum_{i=1}^{n} B W i \leq \sum B W s
$$

Where BWi corresponds to the demand for bandwidth by the $\mathrm{N}$ users available in the system.

However, more resources can be assigned according to availability. This over-assignment would be given by equation (3).

$$
\sum_{t=1}^{T} B W t=\sum B W i
$$

Scenario 2: System Resources Minor to Users'

\section{Demands}

Under this scenario, it is necessary to know that not all the demands of the users will be completely satisfied; however, it should be ensured under a fairness criterion that all users will be able to make use of network resources, even if it is not done in the same time slot but in successive slots. Therefore, equation (4) can be inferred.

$$
(x)=\sum_{s=1}^{s}(U s)-\sum_{l=1}^{s}(C s)
$$

Where Us is the transmission probability of an $\mathrm{x}$ user, to whom a cost Cs is assigned; this cost refers to the possibility of having to transmit again in a later time slot due to the limitations of the system resource.

\section{Design model}

The proposed model takes a spectral occupancy database and secondary user bandwidth demands as input variables. Before carrying out the channel selection process, the spectral information processing block adapts the spectral occupancy data for the channel grouping block using the K-Means technique. Then, this non-supervised learning technique performs 15 iterations of clustering, seeking to gather the channels in two clusters with the best characteristics such as: average availability time, signal-to-noise ratio and probability of availability.

The channel selection process not only takes into account the K-Means technique, but also that channel allocation must be fair for all secondary 
users. For this reason, the "Fairness Algorithm" block is included in the model. This block is also used by the MFA-CRN algorithm (Hernandez, Marquez, \& Pedraza, 2016), which tries to generate a fairness of assignment in the bandwidth. It could be defined as a set of active users throughout the assignment mechanism, assigning a resource Rs to each user s so that equation (5) is fulfilled.

$$
\sum_{s \in S} R s \leq R
$$

Where $R$ is the total amount of resources of the system. However, when the resource offer is greater than the demand, equation (6) must be fulfilled.

$$
\sum_{S \in S} R s+\sum_{S \in S} \frac{N}{S}+R e \leq R
$$

Where $\mathrm{N}$ represents the number of surplus resources, and $R e$ is resources reservation.

This implementation must take into account Fair Queueing, which must be applied in a means of communication with resource restrictions. This algorithm is fundamental in the proposed model due to high traffic handling. It is possible that the average bandwidth assignment for each secondary user does not satisfy them $100 \%$, the next time slot will carry out an average of weights for each channel as shown in equation (7).

$$
\frac{\sum_{i=1}^{n} x_{i} w_{i}}{\sum_{i=1}^{n} w_{i}}+\frac{\sum_{i=1}^{n} x_{r} w_{i}}{\sum_{i=1}^{n} w_{i}}
$$

Where xi represents the percentage of channel assignment for elapsed time intervals and wi represents the weight assigned to each of the channels according to the assignments made. In addition, $x r$ represents the percentage of additional channel assignment that arose due to a greater supply of channel demand.

It is possible to adjust the model to make a fair assignment of bandwidth for all secondary users; it is also possible to select channels for the respective allocation with the fair model and the channel grouping algorithm by the K-Means technique.

\section{SIMULATION}

The requested bandwidth for each of the SU was quantified based on the number of channels required according to the type of application developed by each user. These demands can be single channel or multiple channels. For the simulation, 4 types of demands were developed (from the most demanding in resources to the least demanding) in order to perform a performance evaluation of the algorithm proposed in these scenarios. The bandwidth of each channel is $100 \mathrm{KHz}$. The 4 types of demands are shown in table 1.

The $4 \mathrm{SU}$ classes (which demand 10, 4, 2 or 1 channel, respectively) were determined by a transfer rate analysis corresponding to 5 types of services or applications that an SU commonly used today and whose demand is increasing. These services are:

- Streaming Services

- Videoconferencing services

- Web Services

- Voice Services

- Multimedia Services.

Table 1. Types of SU demand

\begin{tabular}{clccccc}
\hline Demand & \multicolumn{1}{c}{ Description } & $\begin{array}{c}\text { Users 10 } \\
\text { Channels }\end{array}$ & $\begin{array}{c}\text { Users 4 } \\
\text { Channels }\end{array}$ & $\begin{array}{c}\text { Users 2 } \\
\text { Channels }\end{array}$ & $\begin{array}{c}\text { Users 1 } \\
\text { Channel }\end{array}$ & Total Users \\
\hline A & Strong over-assignment & 5 & 5 & 10 & 20 & 40 \\
\hline B & Mild over-assignment & 10 & 10 & 15 & 30 & 65 \\
\hline C & Mild Punishment & 25 & 15 & 25 & 35 & 100 \\
\hline D & Strong Punishment & 40 & 15 & 20 & 25 & 100 \\
\hline
\end{tabular}

Source: own the author 


\section{RESULTS}

Measurements of bandwidth, average delay, failed handoffs, handoffs and throughput were performed for 4 different types of demands in a time range of 10 minutes.

Table 2 shows the way the demands are classified.

Table 2. Types of analyzed demands

\begin{tabular}{ccc}
\hline Type & Number of users & Supply/demand ratio \\
\hline DMA & 40 & $240 \%$ \\
\hline DMB & 65 & $132 \%$ \\
\hline DMC & 10 & $67 \%$ \\
\hline DMD & 10 & $50 \%$ \\
\hline
\end{tabular}

Source: own the author

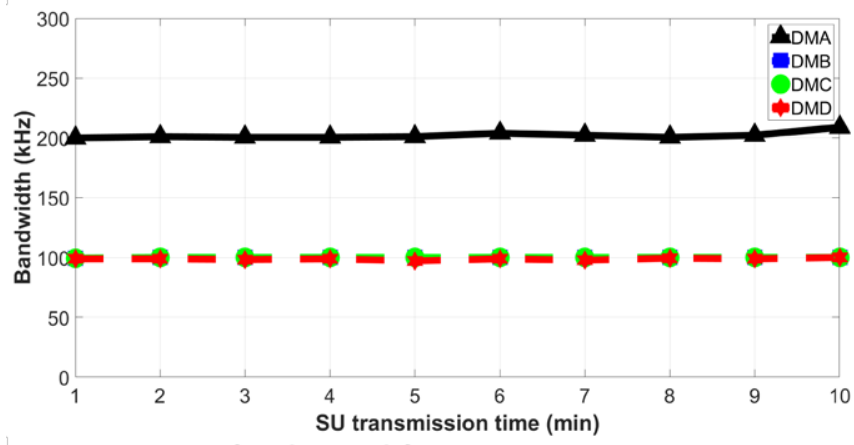

a. AB of 1 channel for DMA,DMB,DMC y DMD

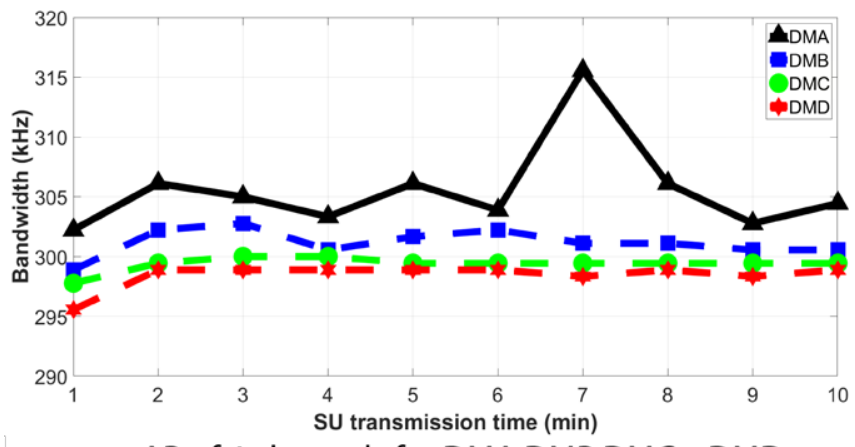

c. $A B$ of 4 channels for $D M A, D M B, D M C$ y DMD
The results are presented through figures 2 to 6 .

Figure 2 states that the bandwidth assignment is more effective in DMA and DMB for 1 channel and 2 channels; however, the channel assignment was approximately $25 \%$ less to what was requested for the case of 4 channels (figure 2c), and the allocation was between $40 \%$ and $80 \%$ compared to the required in the case of 10 channels (figure $2 d$ ). The factor to keep in mind is the number of users who are requesting channels.

Regarding the delay, it is observed that the behavior is similar in each type of demand versus the number of channels requested. However, the case of the 10-channel delay (figure 3d) determines that the shortest delay is for DMA (strong over-assignment), which indicates that a larger assignment of

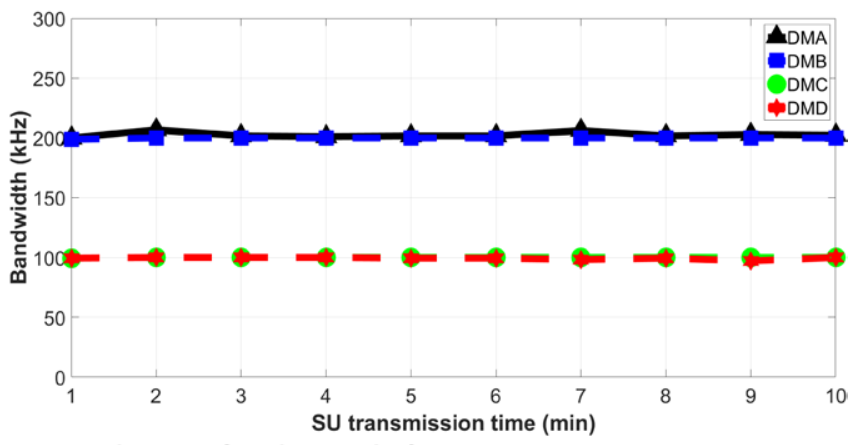

b. AB of 2 channels for DMA,DMB,DMC y DMD

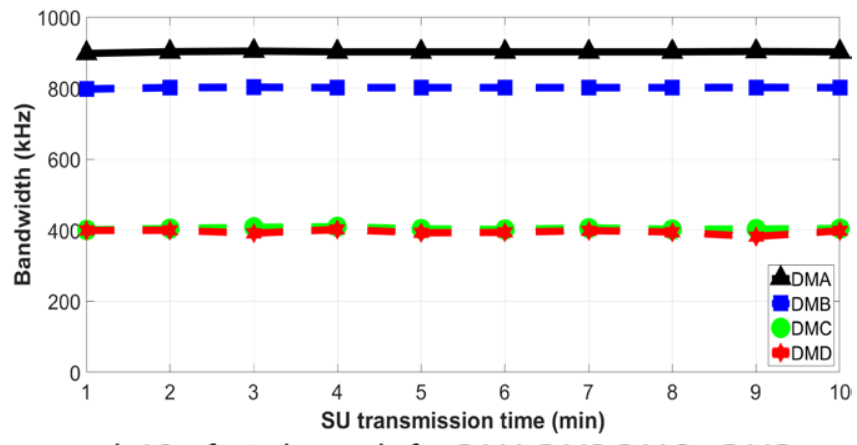

d. $A B$ of 10 channels for DMA,DMB,DMC y DMD

Figure 2. Average bandwidth in high traffic for DMA, DMB, DMC and DMD

Source: own the author 


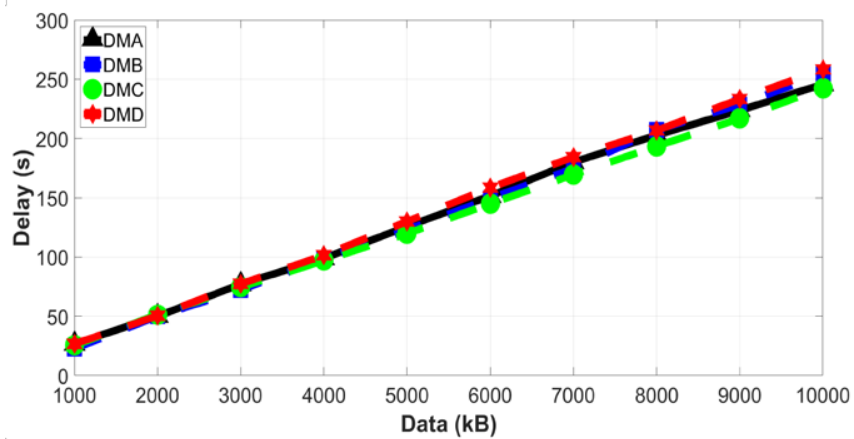

a. Delay of 1 channel for DMA,DMB,DMC and DMD

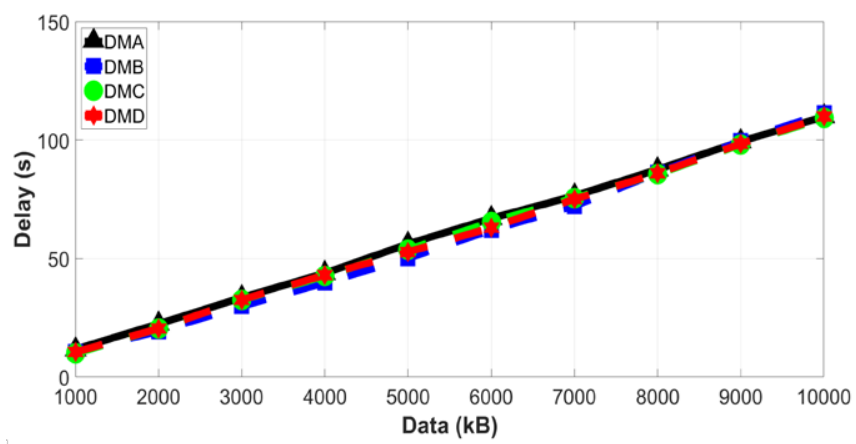

c. Delay of 4 channels for DMA,DMB,DMC and DMD

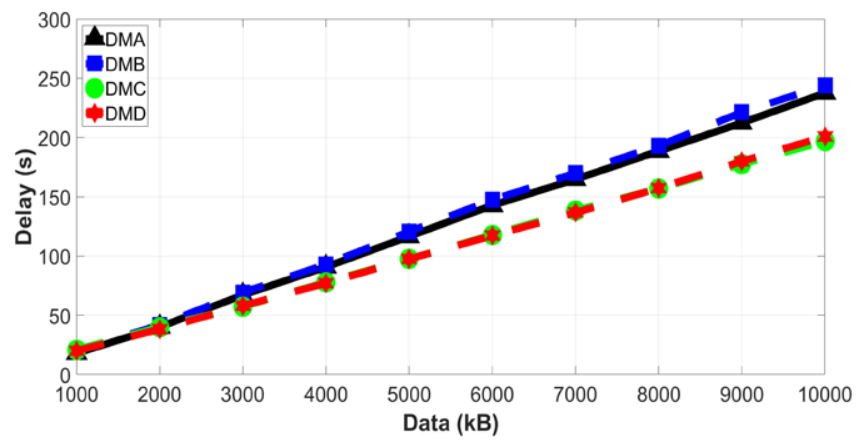

b. Delay of 2 channels for DMA,DMB,DMC and DMD

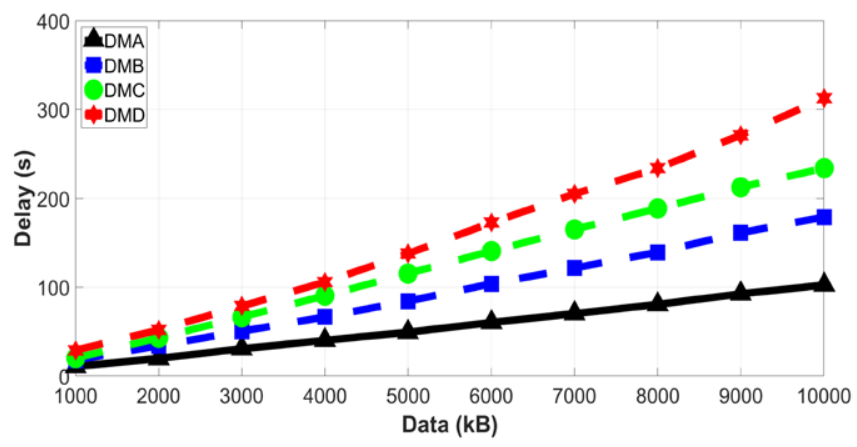

d. Delay of 10 channels for DMA,DMB,DMC and DMD

Figure 3. Average delay in high traffic for DMA, DMB, DMC and DMD

Source: own the author

channels to each user will allow the generated delay in the transmission to be positively affected.

Figure 4 shows an increasing in the number of failed handoffs for DMA for 1-, 2-, 4- and 10-channels demand because the algorithm searches for a greater number of channels against what is requested to generate an over-assignment. Although this may look as a negative aspect, it is observed that the delay in transmission (figure 3 ) is reduced, and there is a significant increase in throughput (figure 6).

The same occurs in the case of failed handoffs (figure 5). It can be observed that for DMA the number of handoffs was relatively superior to the other types of demands, although it was the one that had better throughput for each type of demand and each type of user (see figure 6). 


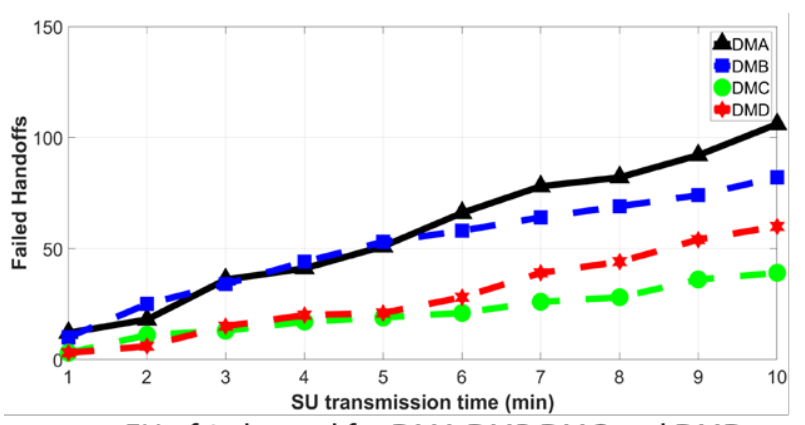

a. FH of 1 channel for DMA,DMB,DMC and DMD

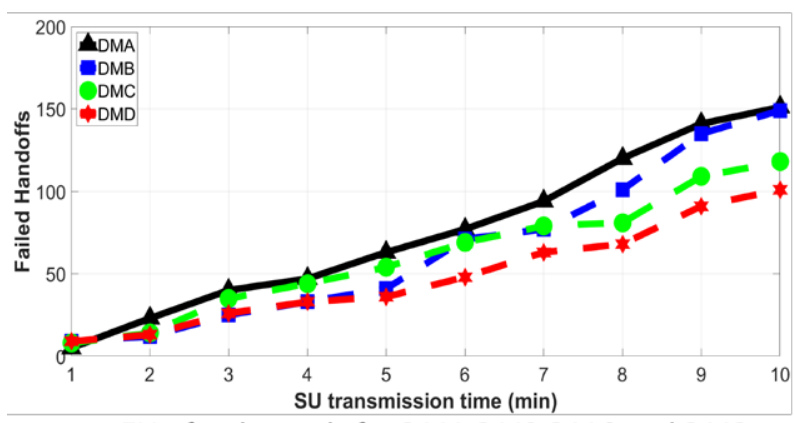

c. FH of 4 channels for DMA,DMB,DMC and DMD

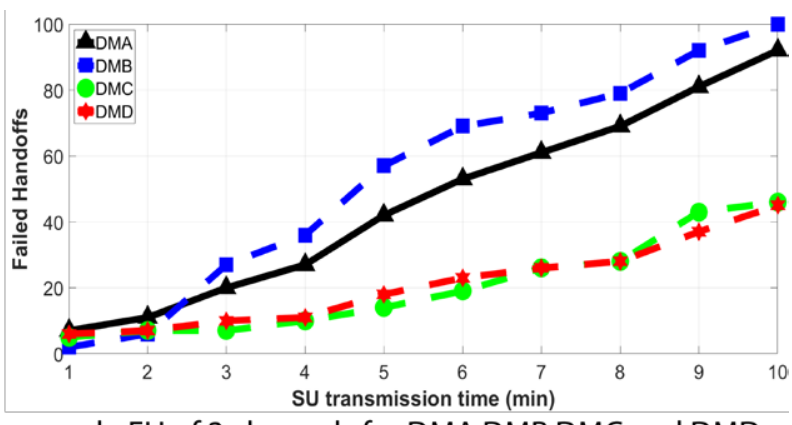

b. FH of 2 channels for DMA,DMB,DMC and DMD

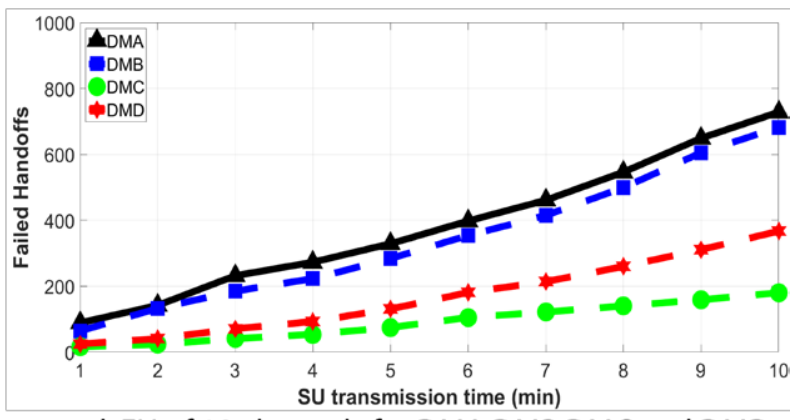

d. FH of 10 channels for DMA,DMB,DMC and DMD

Figure 4. Failed Handoffs in high traffic for DMA, DMB, DMC and DMD

Source: own the author

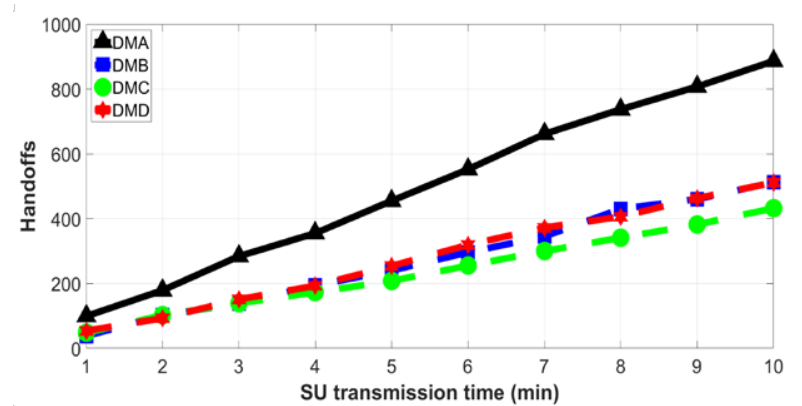

a. SH of 1 channel for DMA,DMB,DMC and DMD

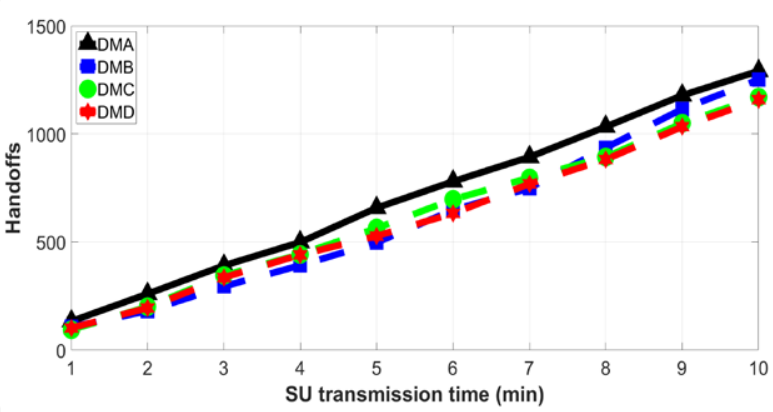

c. SH of 4 channels for DMA,DMB,DMC and DMD

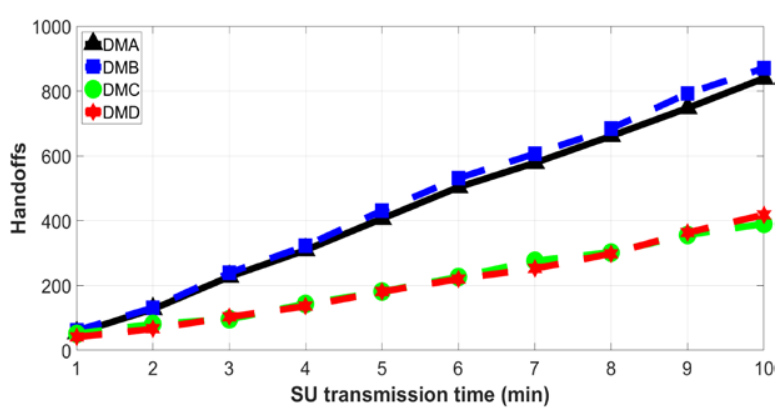

b. SH of 2 channels for DMA,DMB,DMC and DMD

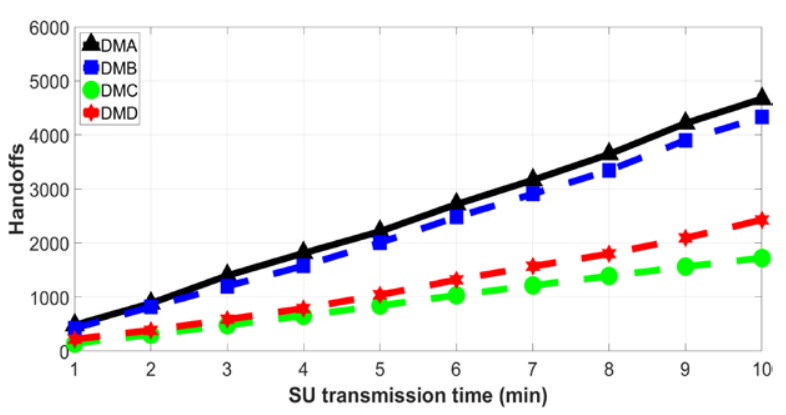

d. SH of 10 channels for DMA,DMB,DMC and DMD

Figure 5. Handoffs in high traffic for DMA, DMB, DMC and DMD

Source: own the author 


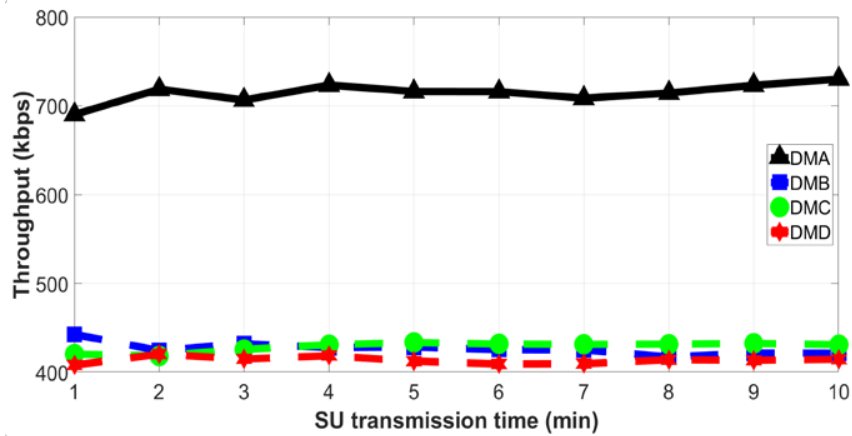

a. TH of 1 channel for DMA,DMB,DMC and DMD

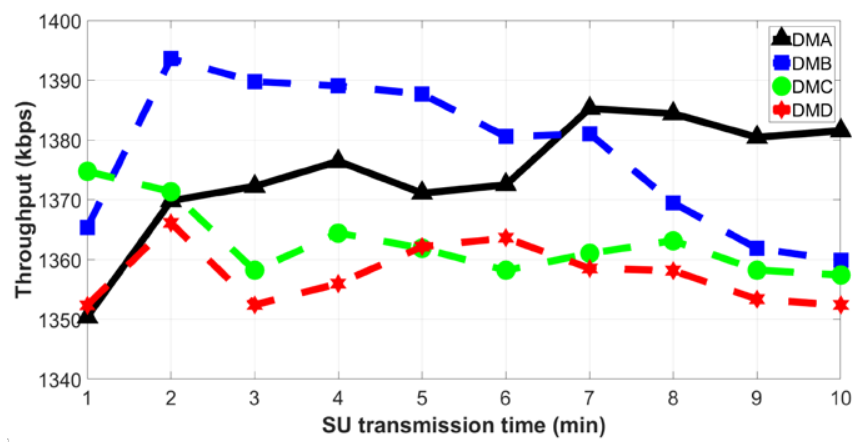

c. TH of 4 channels for DMA,DMB,DMC and DMD

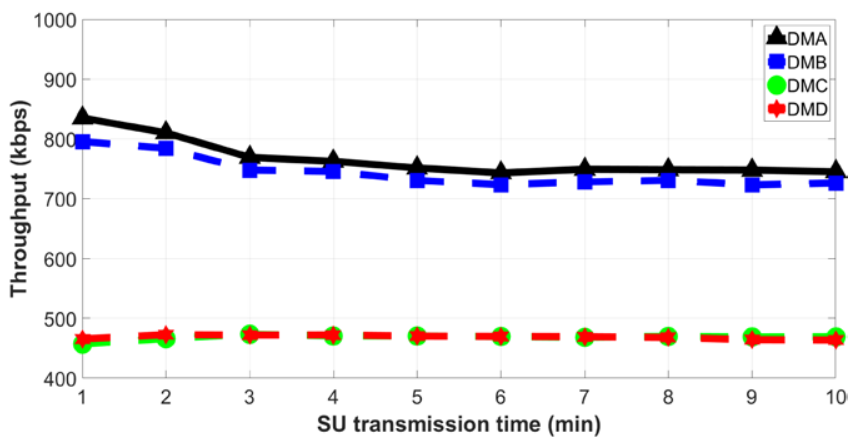

b. TH of 2 channels for DMA,DMB,DMC and DMD

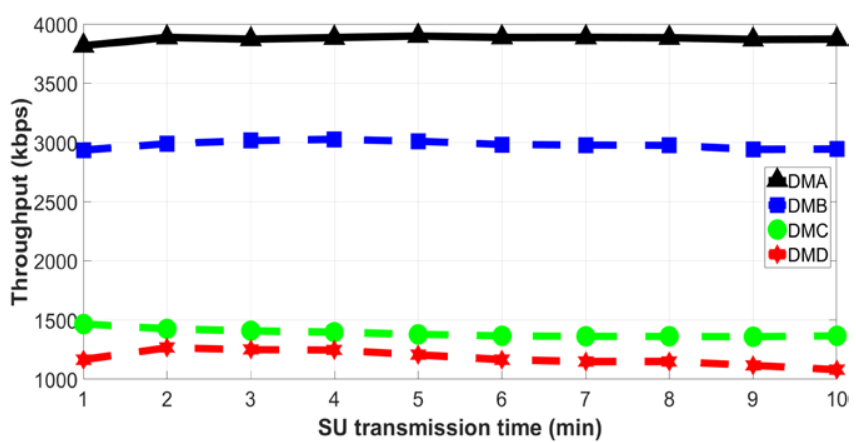

d. TH of 10 channels for DMA,DMB,DMC and DMD

Figure 6. Throughput in high traffic for DMA, DMB, DMC and DMD

Source: own the author

\section{CONCLUSIONES}

It is observed that the number of failed handoffs increased in the over-assignment scenarios because the algorithm tries to maintain this over-assignment of channels for the secondary users in the network.

The mean time availability, signal-to-noise ratio and availability probability criteria selected as K-Means grouping criteria were able to generate 2 channel clusters with the best weights that allowed significant throughput for DMA and DMB and also allowed having outstanding performance for DMC and DMD.

In spite of the increasing in the number of handoffs, results show that metrics such as average bandwidth, average throughput and average delay are never negatively impacted by this increasing in the number of handoffs.

\section{ACKNOWLEDGEMENTS}

The authors wish to thank Colciencias and the District University Francisco José de Caldas, for the supporting and funding during the course of this research project.

\section{REFERENCES}

Bajali, V., Nagendra, T., Hota, C., \& Raghurama, G. (2016). Cooperative spectrum sensing in Cognitive Radio: An Archetypal Clustering approach. Wireless Communications, Signal Processing and Networking (WiSPNET) (pp. 1137-1143). IEEE.

Chen, C., Wei, X., Jin, Y., \& Ge, S. (2013). A Delay Sensitive Time Slots Scheduling in Vehicular Sensor Networks. Intelligent Networking and Collaborative Systems (INCoS) (pp. 448-455). IEEE. 
Chen, X., Hou, F., Huang, H., \& Jing, X. (2016). Principle component analysis based cooperative spectrum sensing in cognitive radio. Communications and Information Technologies (ISCIT) (pp. 602605). IEEE.

Hernandez, C., Marquez, H., \& Pedraza, L. (2016). Multichannel Allocation Spectrum Model with Fairness Criterion for Cognitive Radio Networks. Contemporary Engineering Sciences, 9(31), 1503-1524.

Hernández, C., Márquez, H. \& Pedraza, L. (2017). Modelo de asignación multicanal con equidad para la movilidad espectral en redes de radio cognitiva. Bogotá, Colombia: Editorial UDistrital.

Hernández, C., Páez, I. \& Giral, D. (2017). Modelo adaptativo multivariable de handoff espectral para incrementar el desempeño en redes móviles de radio cognitiva. Bogotá, Colombia: Editorial UDistrital.

Kang, S., Ryu, J., Lee, J., \& Jeong, J. (2011). Analysis of space-time adaptive processing performance using K-means clustering algorithm for normalisation method in non-homogeneity detector process. IET signal processing, 113-120.

Kumar, V., Kandpal, D. C., Jain, M., Gangopadhyay, R., \& Debnath, S. (2016). K-mean clustering based cooperative spectrum sensing in generalized $\kappa-\mu$ fading channels. Communication (NCC), 2016 Twenty Second National Conference, (pp. 1-5).

Lu, Y., Zhu, P., Wang, D., \& Fattouche, M. (2016). Machine learning techniques with probability vector for cooperative spectrum sensing in cognitive radio networks. Wireless Communications and Networking Conference (WCNC) (pp. 1-6). IEEE.

McLean, R. K., Silvius, M. D., \& Hopkinson, K. M. (2013). Method for Evaluating k-Means Clustering for Increased Reliability in Cognitive Radio Networks. Software Security and Reliability (SERE), 2013 IEEE 7th International Conference (pp. 99108). IEEE.
Moumena, A. (2015). Abnormal behavior detection of jamming signal in the spectrum using a combination of compressive sampling and intelligent bivariate k-means clustering technique in wideband cognitive radio systems. 4th International Conference on Electrical Engineering (ICEE) (pp. 1-4). IEEE.

Nguyen, D. D., Madhukumar, A. S., Tio, S. D., \& Ng, B. C. (2010). Cognitive Radios In Cooperative Environment:Detection, Sensing and Clustering of Spectral Bands. Vehicular Technology Conference Fall (VTC 2010-Fall) (pp. 1-5). IEEE.

Pandey, R. (2015). Elective Recommendation Support through K-Means Clustering Using R-Tool. Computational Intelligence and Communication Networks (CICN) (pp. 851-856). IEEE.

Ramler, I. P. (2008). Improved statistical methods for $k$-means clustering of noisy and directional data. Proquest.

Tragos, E. Z., Zeadally, S., Fragkiadakis, A. G., \& Siris, V. A. (2013). Spectrum assignment in cognitive radio networks: A comprehensive survey. IEEE Communications Surveys \& Tutorials, 13(3), 1108-1138.

Vassilvitskii, S. (2007). K-Means: algorithms, analyses, experiments. Stanford University.

Xu, J., Han, J., \& Nie, F. (2016). Discriminatively embedded k-means for multi-view clustering. Conference on Computer Vision and Pattern Recognition (pp. 5356-5364). IEEE.

Xue, H., \& Feifei, G. (2015). A machine learning based spectrum-sensing algorithm using sample covariance matrix. Communications and Networking in China (ChinaCom), 2015 10th International Conference (pp. 476-480). IEEE.

Zhang, S., Hu, J., Bao, Z., \& Wu, J. (2013). Prediction of spectrum based on improved RBF neural network in cognitive radio. International Conference on Wireless Information Networks and Systems (WINSYS).

\section{(C) $(1) \Theta$}

